\title{
Mistrust and Mandates: COVID-19 Vaccination in the Military
}

Because of some things that have happened in the past, there's a degree of mistrust,
and I think we have to collectively work hard to dispel rumors and to provide facts to people.
Secretary of Defense Lloyd Austin ${ }^{1}$

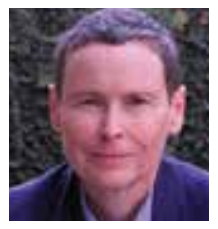

Cynthia Geppert is Editor-in-Chief; Chief, Consultation Psychiatry and Ethics, New Mexico VA Health Care System; and Professor and Director of Ethics Education at the University of New Mexico School of Medicine in Albuquerque. Correspondence: Cynthia Geppert (ethicdoc@comcast.net)

Fed Pract. 2021;38(6) Published online June 10 doi:10.12788/fp.0143 li $t$ is June and most of us are looking forward to a more normal summer than the one we had in 2020. Many Americans have been vaccinated and states are rolling back some (or all) masking requirements and restrictions on gatherings. In many sectors, including the US Department of Defense (DoD) and the US Department of Veterans Affairs (VA), worries from public health officials about vaccine supply and how to ethically allocate demand have given way to a new set of concerns: We have the shots, but for widespread protection we have to get them into arms.

The reluctance to roll up the sleeve is known as vaccine hesitancy. The National Academies of Science comments on vaccine hesitancy in its report on COVID-19 vaccination allocation. "Potential consequences of vaccine hesitancywhich the committee views as an attitude, preference, or motivational state-are the behaviors of vaccine refusal or delay."

On that count, there was encouraging albeit unexpected news in waning days of May. Media reported a sharp increase in the COVID-vaccination of military personnel. Unnamed DoD officials indicated, they had seen a $55 \%$ increase in the vaccination of active-duty service members over the previous month. This news represents a dramatic turnaround in a trend of vaccine hesitancy among military members that has persisted since the vaccine became available. ${ }^{3}$ Even last month, this would have been a very different column. The DoD has not disclosed the exact number of service members who have declined COVID19 vaccination but multiple news outlets have documented that there was widespread and significant vaccine hesitancy among military personnel. In February, Military News reported that one-third of troops who were offered the vaccine declined it; and in April, USA Today stated that $40 \%$ of Marines had refused vaccination. ${ }^{4,5}$
Still, it is worth examining the data on vaccination among active duty service members. From December 2020 through March 2021, the military conducted the first study to evaluate rates of vaccine initiation and completion in the military in general and for service members from racial/ethnic minorities in particular. Black military personnel were $28 \%$ less likely than non-Hispanic White service members to initiate vaccination against coronavirus even after adjusting for other possible confounders. Just $29 \%$ of White, $25.5 \%$ of Hispanic, and $18.7 \%$ of Black service members had initiated the vaccine process in the survey. ${ }^{6}$

The authors suggest that in part, vaccine hesitancy explains the findings. ${ }^{4}$ Vaccine hesitancy among racial and ethnic minorities is even more tragic because these same already disadvantaged cohorts have disproportionately suffered from COVID-19 throughout the pandemic with higher rates of infection, serious illness requiring hospitalization, and infectionrelated morbidity. ${ }^{7}$

Vaccine hesitancy, delay, or refusal in Black Americans whether military or civilian often is attributed to the historical abuses like the Tuskegee syphilis experiments or the more recent example of cancer cell lines taken from Henrietta Lacks without consent. ${ }^{8}$ Such government sponsored betrayals no doubt are the soil in which hesitancy grows but recent commentators have opined that focusing solely on these infamous examples may ignore current systemic racism that is pervasively feeding Black Americans reluctance to consider or accept COVID-19 vaccination. ${ }^{9}$ Blaming infamous research also provides a convenient excuse for confronting contemporary racial discrimination in health care and taking responsibility as health care practitioners for reversing it. "Framing the conversation about distrust in COVID vaccines in terms of everyday racism rather 
than historical atrocities may increase underserved communities' willingness to be vaccinated," Bajaj and Stanford wrote in a recent recent New England Journal of Medicine commentary. "When we hyperfocus on Sims, Lacks, and Tuskegee, we ascribe the current Black health experience to past racism, rooting our present in immovable historical occurrences and undermining efforts to combat mistrust. Everyday racism, by contrast, can be tackled in the present." 9

The study of racial/ethnic disparities in COVID-19 vaccination in active-duty service members was a work product of the Armed Forces Health Surveillance Division. The authors underscore several factors that support the connection between discrimination and vaccine hesitancy in the military. Lack of access to and ability to obtain COVID-19 vaccination continues to be a major barrier that disadvantaged populations must overcome. ${ }^{10}$ The COVID-19 vaccine is widely available, easily obtained, and free of charge for all military personnel. Yet the vaccine hesitancy in the military parallels that of the civilian sector. This led the study authors to opine that, "forces external to the U.S. Military, such as interpersonal and societal factors also contribute to vaccine hesitancy among military service members."

Obviously, any unvaccinated active-duty service member reduces the combat readiness of the fighting force a consideration that led some in Congress to call for mandating vaccination. The vaccine is currently being administered under an emergency use authorization (EUA), which prevents even the military from mandating it. ${ }^{11}$ Even if President Joseph Biden obtained a waiver to make the vaccine mandatory, the implications of forcing service members who have volunteered to serve their country is ethically problematic. Those problems are exponentially amplified when applied to members of ethnic and racial minorities who have a past and present of health disparities and discrimination. Respecting the decision of those in uniform to decline COVID-19 vaccination is the first and perhaps most important step to rebuilding the trust that is the most promising means of reducing vaccine hesitancy.
Part of the accountability we all bear for health care inequity and racism is to continue the work of this landmark study to better understand vaccine hesitancy among military and veteran cohorts, develop counseling and education that target those attitudes, beliefs, and motivations with education, counseling, and support. All of us can in some small measure follow the ethical mandate "to dispel rumors and provide facts to people" of Secretary Austin, a Black retired 4-star Army general. ${ }^{1}$

\section{Disclaimer}

The opinions expressed herein are those of the authors and do not necessarily reflect those of Federal Practitioner, Frontline Medical Communications Inc., the US Government, or any of its agencies.

\section{References}

1. Garmone J. Secretary of Defense Addresses Vaccine Hesitancy in the Military. Published February 25, 2021. Accessed May 26, 2021. https://www.defense.gov/Explore/News/Article/ Article/2516511/secretary-of-defense-addresses-vaccine-hesitancy-in-military/

2. National Academies of Sciences, Engineering, and Medicine. Framework for Equitable Allocation of COVID-19 Vaccine. The National Academies of Science; 2020:188. doi:10.17226/25917

3. Liebermann O. US military sees $55 \%$ jump in COVID-19 vaccinations over last month. Published May 20, 2021. Accessed May 26, 2021. https://www.cnn.com/2021/05/20/politics /us-military-covid-vaccinations/index.html

4. Kime P. Almost one-third of us troops are refusing COVID-19 vaccines, officials Say. Published February 17, 2021. Accessed May 26, 2021. https://www.military.com/daily-news/2021/02/17 /almost-one-third-of-us-troops-are-refusing-covid-vaccines -officials-say.html

5. Elbeshbishi S. Nearly $40 \%$ of Marines decline COVID-19 vaccine, prompting some Democrats to urge Biden to set mandate for the military. USA Today. April 10, 2021. Accessed May 26, 2021. https://www.usatoday.com/story/news /politics/2021/04/10/covid-vaccine-nearly-forty-percent-us -marines-decline/7173918002/

6. Lang MA, Stahlman S, Wells NY, et al. Disparities in COVID-19 vaccine initiation and completion among active component service members and health care personnel, 11 December 2020-12 March 2021. MSMR. 2021;28(4):2-9.

7. Webb Hooper M, Nápoles AM, Pérez-Stable EJ. COVID-19 and racial/ethnic disparities. JAMA. 2020;323(24):2466-2467. doi:10.1001/jama.2020.8598

8. Kum D. Fueled by a history of mistreatment, Black Americans distrust the new COVID-19 vaccines. TIME. December 8, 2020. Accessed May 26, 2021.https://time.com/5925074/black -americans-covid-19-vaccine-distrust/

9. Bajaj SS, Stanford FC. Beyond Tuskegee - Vaccine Distrust and Everyday Racism. N Engl J Med. 2021;384(5):e12. doi:10.1056/NEJMpv2035827

10. Feldman N. Why Black and Latino people still lag on COVID19 vaccines-and how to fix it. NPR. April 26, 2021. Accessed May 26, 2021. https://www.npr.org/sections/health -shots/2021/04/26/989962041/why-black-and-latino-people -still-lag-on-covid-vaccines-and-how-to-fix-it

11. Kaufman E. Lawmakers ask Biden to issue waiver to make COVID-19 vaccination mandatory of members of the military. Updated March 24, 2021. Accessed May 26, 2021. https://www .cnn.com/2021/03/24/politics/congress-letter-military-vaccine /index.html 